\title{
Criminologie
}

\section{L’adhésion à la culture de gang}

Le rôle de l'identité ethnique chez les jeunes contrevenants de Montréal et ses environs

\section{Allegiance to gang culture}

\author{
The role of ethnic identity among young offenders in the \\ Montreal area
}

\section{La adhesión a la cultura de pandilla} El rol de la identidad étnica en los jóvenes delincuentes de
Montreal y alrededores

\section{Manuelle Bériault, Catherine Laurier et Jean-Pierre Guay}

Volume 50, numéro 1, printemps 2017

URI : https://id.erudit.org/iderudit/1039805ar

DOI : https://doi.org/10.7202/1039805ar

Aller au sommaire du numéro

\section{Éditeur(s)}

Les Presses de l’Université de Montréal

\section{ISSN}

0316-0041 (imprimé)

1492-1367 (numérique)

Découvrir la revue

Citer cet article

Bériault, M., Laurier, C. \& Guay, J.-P. (2017). L’adhésion à la culture de gang : le rôle de l'identité ethnique chez les jeunes contrevenants de Montréal et ses environs. Criminologie, 50(1), 287-310. https://doi.org/10.7202/1039805ar
Résumé de l'article

La présence accrue de jeunes faisant partie de groupes ethnoculturels minoritaires dans les institutions pour jeunes contrevenants au Québec est une problématique complexe et préoccupante. Cette étude explore le rôle de l'identité ethnique et du fait de faire partie d'un groupe de minorités racisées dans l'association autorévélée à un gang de rue et dans l'adhésion à la culture de gang. Les participants ( $\mathrm{n}=69$; âge 14-20 ans) ont été recrutés dans des centres de réadaptation de la région montréalaise. Il ressort des résultats que le fait de se reconnaître membre d'un gang de rue ne diffère pas entre les jeunes issus des minorités racisées et ceux qui n'en sont pas issus. Les analyses de régressions multiples effectuées révèlent que plus un jeune contrevenant rapporte un niveau d'exploration de l'identité ethnique élevé, plus il adhère aux dimensions signes et symboles et règles et rituels de l'adhésion à la culture de gang, et ce, peu importe son âge ou qu'il fasse partie d'un groupe de minorités racisées. Cette étude fait ressortir l'importance de s'intéresser aux questions identitaires lors d'interventions auprès de jeunes contrevenants, et ce, peu importe leurs origines.
Tous droits réservés @ Les Presses de l’Université de Montréal, 2017

Ce document est protégé par la loi sur le droit d'auteur. L'utilisation des services d'Érudit (y compris la reproduction) est assujettie à sa politique d'utilisation que vous pouvez consulter en ligne.

https://apropos.erudit.org/fr/usagers/politique-dutilisation/ 


\title{
L'adhésion à la culture de gang Le rôle de l'identité ethnique chez les jeunes contrevenants de Montréal et ses environs ${ }^{1}$
}

\author{
Manuelle Bériault \\ Doctorat en psychologie (D. Psy) \\ Cheminement en enfance et en adolescence \\ Université de Sherbrooke \\ manuelle.beriault@usherbrooke.ca \\ Catherine Laurier ${ }^{2}$ \\ Professeure associée \\ École de criminologie, Université de Montréal \\ Chercheure, Institut universitaire Jeunes en difficulté \\ catherine.laurier@cjm-iu.qc.ca \\ Jean-Pierre Guay \\ Professeur agrégé \\ École de criminologie, Université de Montréal \\ Chercheur, Centre international de criminologie comparée \\ jean-pierre.guay@umontreal.ca
}

RÉSUMÉ - La présence accrue de jeunes faisant partie de groupes ethnoculturels minoritaires dans les institutions pour jeunes contrevenants au Québec est une problématique complexe et préoccupante. Cette étude explore le rôle de l'identité ethnique et du fait de faire partie d'un groupe de minorités racisées dans l'association autorévélée à un gang de rue et dans l'adhésion à la culture de gang. Les participants ( $\mathrm{n}=69$; âge 14-20 ans) ont été recrutés dans des centres de réadaptation de la région montréalaise. Il ressort des résultats que le fait de se reconnaître membre d'un gang de rue ne diffère pas entre les jeunes issus des minorités racisées et ceux qui n'en sont pas issus. Les analyses de régressions multiples effectuées révèlent que plus un jeune

1. Cette étude a été réalisée avec l'aide financière des Fonds québécois pour la recherche sur la société et la culture (FQRSC) grâce à une subvention octroyée notamment à Catherine Laurier, $\mathrm{Ph}$. D. De plus, cette étude a été soutenue financièrement par des bourses (soutien financier et fin de parcours universitaire) offertes par l'Institut universitaire Jeunes en difficulté, ainsi que par le Centre international de criminologie comparée (CICC), à Manuelle Bériault, B. Sc.

2. Institut universitaire Jeunes en difficulté, 1001, boul. De Maisonneuve Est, Montréal (Québec), Canada, H2L 4R5.

Criminologie, vol. 50, $\mathrm{n}^{\circ} 1$ (2017) 
contrevenant rapporte un niveau d'exploration de l'identité ethnique élevé, plus il adhère aux dimensions signes et symboles et règles et rituels de l'adhésion à la culture de gang, et ce, peu importe son âge ou qu'il fasse partie d'un groupe de minorités racisées. Cette étude fait ressortir l'importance de s'intéresser aux questions identitaires lors d'interventions auprès de jeunes contrevenants, et ce, peu importe leurs origines.

MoTS CLÉs - Identité ethnique, minorités, ethnicité, délinquance juvénile, gang de rue.

\section{Introduction}

Lorsque les études scientifiques se penchent sur les questions liées à la délinquance juvénile et aux gangs de rue, l'accent est généralement porté sur l'identification de groupes ethniques plus à risque de s'associer à un gang (Esbenson et Carson, 2012; Esbensen et Winfree, 1998; Guerra et Smith, 2006; van Gemert, Peterson et Lien, 2008; Wortley et Tanner, 2006). De plus en plus de recherches soulignent l'hétérogénéité de la composition ethnique des gangs et la présence de membres de gang «blancs» (Chatterjee, 2006; Hamel, Alain, Messier-Newman, Domond et Pagé, 2013 ; Wortley et Tanner; 2008). Néanmoins, l'association à un gang de rue est encore perçue comme un phénomène exclusif aux groupes ethnoculturels minoritaires (Perreault et Bibeau, 2003 ; Spergel, 2009), sans toutefois préciser le rôle de l'ethnicité et de la culture ainsi que leurs impacts sur le mode de vie de gang (De laco, 2006), ce qui constitue une lacune importante.

L'objectif général de cet article est d'explorer, auprès de jeunes contrevenants de la région montréalaise et environnante, la contribution propre à l'identité ethnique ${ }^{3}$ et le fait de faire partie d'un groupe de minorités racisées ${ }^{4}$ par rapport à l'association autorévélée à un gang de rue et à l'adhésion de jeunes contrevenants à la culture de gang.

3. Il n'existe aucun consensus dans les études sur l'immigration et les rapports ethniques quant à la définition et à l'utilisation des concepts de race, d'origine ethnique, de minorités visibles ou raciales ou encore d'identité ethnique ou raciale (Rivas-Drake et al., 2014). Par exemple, certains chercheurs incluent le concept d'identité raciale à l'intérieur même du concept d'identité ethnique (Phinney, 1990). D'autres chercheurs y voient plutôt des processus distincts (Rivas-Drake et al., 2014) et préfèrent parler d'identité ethnique et raciale (ERI). L'utilisation du concept d'identité ethnique a été choisie puisqu'il réfère directement au concept mesuré par le Multigroup Ethnic Identity Measure - Revised (MEIM-R) (Phinney et Ong, 2007), employé dans cette étude.

4. Le terme «minorités racisées» a été préféré à celui de «minorités raciales» afin de refléter, non pas des différences sur le plan biologique, comme le suggère l'utilisation du terme «race», mais plutôt de mettre l'accent sur le vécu et les effets sociologiques découlant des expériences de faire partie d'un groupe de personnes qui n'ont pas la peau blanche (Bolafi, Bracalenti, Braham et Gindro, 2003; Mc Andrew, Ledent et Bakshaei, 2013; Mock, 2005). 


\section{Culture de gang}

Un des constats partagés par les chercheurs étudiant le phénomène des gangs de rue est qu'il n'existe aucun consensus quant aux définitions des concepts de «gang de rue» et de «membre de gang» ainsi qu'aux méthodes servant à identifier les personnes associées à ces groupes (Hamel et al., 2013). Toutefois, l'influence des caractéristiques personnelles et sociales des délinquants sur leur association aux gangs de rue ainsi que le fait que les membres de gang commettent un grand nombre de crimes est à ce jour bien documenté dans la littérature scientifique (Hill, Howell, Hawkins et Battin-Pearson, 1999; Klein et Maxson, 2006; Thornberry, Krohn, Lizotte, Smith et Tobin, 2003). Dans les écrits scientifiques, les membres de gangs sont décrits comme des jeunes faisant partie d'une catégorie culturelle à part entière qui favorise l'adoption et le maintien de conduites délinquantes et violentes (Fredette et Guay, 2014). Malgré la mention par plusieurs chercheurs qu'il existe une culture spécifique expliquant l'effet des gangs sur les jeunes en situation de vulnérabilité qui les côtoient (Hagedorn, 2008; Klein, 1995 ; Thorberry et al., 2003), l'influence de cette culture sur la délinquance des jeunes a été peu étudiée (Fredette, 2014). Pour remédier à cette limite, Fredette (2014) a cherché à opérationnaliser et à préciser le concept de culture de gang. À la lumière de ses travaux, la culture de gang est définie comme «un univers de significations partagées, transmis de génération en génération, qui inclut des symboles et des signes de reconnaissance, des règles et des rituels, de même que des normes et des valeurs» (Fredette et Guay, 2014, p. 155). Ainsi, l'adhésion à la culture de gang fait référence à l'utilisation d'attributs visibles (p. ex., nom de groupe, surnom de membre, habillement, graffitis, signes de mains) permettant aux membres de se faire connaître, de se reconnaître et de communiquer entre eux (Hagedorn, 2008; SanchezJancowski, 1991; Savelli, 2004). De son côté, l'adhésion à des règles et à des rituels s'articule autour de prescriptions qui régissent la vie de groupe, de même qu'à l'ensemble des habitudes transmises aux plus jeunes par les membres vétérans (Hagedorn, 2008). On y retrouve entre autres la présence de rituels initiatiques (Vigil, 1988) ou l'usage de la violence, que ce soit pour commettre des délits, défendre un marché criminel, se protéger de groupes rivaux ou encore établir son autorité auprès des pairs (Sanchez-Jankowski, 2003). Finalement, les normes et les valeurs réfèrent à ce qui est posé comme vrai et important et qui sert de principe moral au gang, par exemple l'honneur, la suprématie 
masculine, la loyauté, la cohésion, l'argent et les gains matériels (Cohen, 1955; Hagedorn, 2008; Vigil, 1988).

\section{Minorités ethnoculturelles et gang de rue}

L'Enquête policière canadienne de 2002 (Chettleburgh, 2003) estime que sur l'ensemble du territoire canadien, environ le tiers (36\%) des gangs répertoriés sont constitués d'au moins deux groupes raciaux ou ethniques. La majorité des gangs serait homogène quant aux caractéristiques ethniques de leurs membres. Au Québec, sur le territoire des quatre agences policières ayant répondu à l'enquête ${ }^{5}$, les Noirs constituent le groupe racial le plus représenté dans les gangs de jeunes (51\%), suivi des Blancs $(21 \%)$ et des Latinos-Américains (12\%). Tout comme aux États-Unis (Esbenson et Carson, 2012; Esbenson, Peterson, Taylor et Freng, 2010; Guerra et Smith, 2006; van Gemert et al., 2008), une prédominance des groupes ethnoculturels minoritaires est présente dans les gangs de rue. Pour tenter d'expliquer cette surreprésentation, la littérature actuelle montre que l'adhésion aux gangs est déterminée en grande partie par la précarité des conditions de vie des jeunes issus de groupes ethnoculturels minoritaires (Egley, Howell et Major, 2006), leur isolement, leur marginalisation ou des expériences de discrimination (Chatterjee, 2006; Fredette, 2014; Hamel et al., 2013). Un sentiment d'exclusion relativement aux ressources et aux services de la société pourrait amener les jeunes à créer des gangs ou à s'y joindre aux fins d'accès de manière marginale à ces mêmes buts (Agnew, 1992). Les chercheurs soulignent que l'association à un gang ne serait pas exclusive aux jeunes faisant partie d'un groupe ethnoculturel minoritaire (Esbenson et Carson, 2012), mais serait plutôt le reflet du portrait des quartiers dont ils sont issus (Chettleburgh, 2003; Franzese, Covey et Ménard, 2006; Freng et Esbenson, 2007). Vigil (1988) parle d'un processus d'identification temporaire ou sporadique aux gangs de rue chez des jeunes marginalisés par les effets de la pauvreté et de la discrimination raciale ou culturelle, un constat partagé par Wortley et Tanner $(2006$; 2008). Ces chercheurs canadiens ont mené une étude auprès de 3393 étudiants au secondaire de la région de Toronto. Leurs résultats semblent indiquer que la perception d'injustice raciale ${ }^{6}$ a un

5. Montréal, Québec, Gatineau-Hull, Sainte-Julie.

6. L'étude fait référence aux injustices raciales perçues dans différents secteurs, comme les politiques, la recherche de logement, l'éducation ou les opportunités d'emplois. 
effet plus important sur l'association aux gangs que l'identité ethnique à elle seule. Les jeunes qui percevaient des injustices raciales envers leur groupe ethnique, ainsi que des iniquités sociales pouvant en découler, étaient plus enclins à être impliqués dans un gang que ceux qui n'en percevaient pas, et ce, peu importe leurs origines (Wortley et Tanner, 2006). Dans une étude subséquente, ces mêmes auteurs ont mené 125 entrevues qualitatives spécifiquement auprès de jeunes associés à un gang de rue. Leurs résultats soulignent que les jeunes faisant partie d'un groupe racial minoritaire nommaient davantage que leurs homologues blancs des injustices raciales ou des iniquités sociales comme raison de s'être joints à un gang (Wortley et Tanner, 2008).

Il importe de préciser que la majorité des jeunes issus de communautés ethnoculturelles minoritaires qui vivent dans des conditions désavantagées ne deviennent pas systématiquement membres de gangs (Klein, 1995 ; Guerra et Smith, 2006). Ce constat soulève la pertinence de considérer les facteurs de risque ou de protection individuels et psychologiques lorsqu'il est question de mieux comprendre l'association aux gangs de rue.

\section{Identité ethnique}

Tout en se consacrant aux tâches du développement identitaire propres à la période de l'adolescence et partagées par tous les adolescents, le développement d'une identité ethnique représente une étape importante, particulièrement chez les jeunes faisant partie d'un groupe ethnoculturel minoritaire (Érikson, 1972; Phinney, 1990). Ces derniers adoptent des comportements visant à explorer leurs origines ethniques et éventuellement développer un sentiment d'appartenance relativement à celles-ci. Phinney (1990) considère que l'exploration et l'engagement sont deux processus distincts, mais étroitement liés, menant ultimement à la construction de l'identité ethnique. Spécifiquement, l'exploration fait référence à la période d'expérimentation relativement à ses origines ethniques par l'entremise de différentes activités, comme la lecture, l'apprentissage de pratiques culturelles ou encore assister à des évènements portant sur sa culture. Les adolescents peuvent chercher à recueillir des informations concernant leurs origines auprès de sources variées, dont la famille et les pairs. S'associer avec d'autres adolescents qui vivent une recherche identitaire semblable à la leur s'avère une façon de mieux connaître et comprendre leur culture et leurs origines ethniques 
ou raciales (Umaña-Taylor et al., 2014). L'engagement réfère au sentiment d'appartenance ou de fierté envers ses origines ethniques et a son origine dans une connaissance et une compréhension de son identité ethnique provenant du processus d'exploration. Sans exploration, l'engagement peut être moins ancré et plus sujet à changer selon les expériences. Une étude mexicaine de Guitart (2011) rapporte un niveau d'identité ethnique et de recherche identitaire plus élevé chez les adolescents issus du groupe ethnique minoritaire (groupe indigène), lorsque comparés à ceux du groupe ethnique majoritaire (groupe métis). L'identité ethnique prend une importance particulière pour les individus en situation de changement ou de contact avec d'autres groupes ethniques, puisque ces contextes activent une prise de conscience des différences qui séparent les jeunes faisant partie d'un groupe ethnique minoritaire comparativement à ceux de la majorité ethnique (Phinney, 2008). Cependant, ce processus identitaire relativement à ses origines ethniques ou culturelles n'est pas propre aux jeunes appartenant à une minorité ethnoculturelle mais s'applique à tout individu, toutes origines ethniques confondues (Phinney, 1990).

\section{Identité ethnique et gang de rue}

Des études soulignent que les jeunes associés à un gang de rue présentent également un sentiment d'appartenance plus élevé envers leurs origines ethniques que ceux n'y étant pas associés (French, Kim et Pillado, 2006; Knight et al., 2012). L'homogénéité de la composition ethnique de plusieurs gangs peut amener les jeunes membres de ces groupes à s'y associer pour obtenir plus d'informations sur ce que cela signifie d'être d'une certaine origine ethnique, tout en offrant une plateforme pour commettre davantage d'actions délinquantes par l'entremise des activités liées aux gangs. De laco (2006) s'est intéressée à la question de l'identité ethnique et culturelle auprès de jeunes contrevenants montréalais de diverses origines (québécoise, haïtienne, jamaïcaine, latino-américaine et autres) impliqués dans les gangs de rue. Les entrevues qu'elle a menées auprès d'une cinquantaine de jeunes soulignent l'homogénéité de la composition ethnique des gangs dont les jeunes faisaient partie. Ce qui l'amène à poser l'hypothèse que ces jeunes avaient en partie décidé de se joindre à un gang pour côtoyer d'autres jeunes avec un vécu des trajectoires de vie, des expériences culturelles ou raciales semblables aux leurs (p. ex., sentiment d'exclusion ou de discrimination par la société). 
Les études font ressortir l'importance de considérer l'identité ethnique comme s'inscrivant dans un processus d'intégration de croyances chez les jeunes par rapport à leurs origines ethniques, mais également comme découlant de circonstances liées au fait de faire partie d'un groupe de minorités racisées et de partager des expériences culturelles découlant de cette appartenance. Cette brève recension des écrits soulève des questionnements sur lesquels se pencher pour mieux comprendre le rôle de l'identité ethnique et du fait de faire partie d'un groupe de minorités racisées sur l'association aux gangs de rue et l'adhésion à la culture de gang chez les jeunes contrevenants. Les études qui se sont intéressées à la surreprésentation des jeunes faisant partie d'un groupe ethnoculturel minoritaire dans les gangs de rue ont été principalement menées auprès de populations américaines. Qu'en est-il des jeunes contrevenants qui s'associent à un gang de rue à Montréal et dans ses environs? Ces jeunes contrevenants appartiennent-ils à un groupe de minorités racisées comme semblent l'indiquer les écrits scientifiques? À quel niveau ces jeunes se situent-ils en ce qui a trait à l'exploration de leur identité ethnique et à leur engagement envers celle-ci? Diffèrent-ils de leurs homologues qui ne sont pas membres de gang de rue sur cet aspect? De plus, l'adhésion à la culture de gang diffère-t-elle entre les jeunes qui font partie d'une minorité racisée et ceux qui n'en font pas partie? L'objectif principal de cet article est d'explorer la contribution propre à l'identité ethnique et le fait de faire partie d'un groupe de minorités racisées par rapport à l'association à un gang de rue et à l'adhésion à la culture de gang. Plus spécifiquement, cet article vise à (i) étudier la relation entre le niveau d'identité ethnique et l'association autorévélée à un gang, ainsi que l'adhésion à la culture de gang, et (ii) étudier la relation entre le fait de faire partie d'un groupe de minorités racisées et l'association autorévélée à un gang, ainsi que l'adhésion de jeunes contrevenants à la culture de gang. L'opérationnalisation récente du concept de culture de gang permettra d'explorer ces avenues.

\section{Méthodologie}

\section{Participants}

Les participants de cette étude font partie d'un sous-échantillon de trois études plus larges (Fredette, 2014; Guay et al., 2015; Laurier, Guay, 
Lafortune et Toupin, 2015) ${ }^{7}$ menées auprès de jeunes contrevenants pris en charge sous la Loi sur le système de justice pénale pour les adolescents (LSJPA) et d'adultes contrevenants pris en charge par les services correctionnels $(n=260)$. Le recrutement et les passations des questionnaires se sont déroulés conjointement. Seuls les 69 participants pris en charge sous la LSJPA et dont les informations concernant l'identité ethnique et l'adhésion à la culture de gang étaient disponibles ont été retenus. Au moment de la passation, ces 69 jeunes contrevenants étaient âgés de 14 à 20 ans $(M=16,8 ; E ́ T=1,17)$. En ce qui a trait à la nature des crimes autorapportés commis dans les 12 mois avant leur prise en charge, $94 \%$ des jeunes ont déclaré au moins un crime contre la personne (menace, vol qualifié, voies de fait, décharge d'une arme, agression sexuelle); $88 \%$, au moins un crime contre les biens (méfait, incendie criminel, introduction par effraction, vol de plus ou moins $5000 \$$, fraude); et $80 \%$ ont dit avoir participé à du trafic de stupéfiants. Les trois quarts des participants étaient placés en garde ouverte ou fermée alors que le quart étaient suivis dans la communauté. La durée moyenne de prise en charge (incluant la période de mise sous garde ou du suivi en communauté, ainsi que celle de surveillance) était de 16 mois (de 6 à 45 mois).

\section{Procédure}

Le recrutement s'est effectué par un système de référence où les participants étaient dirigés vers les assistants de recherche par les agents de liaison à la recherche des centres jeunesse. À la suite de la présentation du projet de recherche par les assistants de recherche, les jeunes étaient libres d'accepter ou de refuser d'y participer. Le consentement des parents a été obtenu pour les participants mineurs et les participants majeurs donnaient le leur. Les rencontres se sont déroulées dans les bureaux des centres jeunesse. Les critères d'exclusion étaient la présence de déficience intellectuelle ou de troubles psychotiques notés au dossier, et ce, afin de s'assurer que tous étaient en mesure de répondre aux questionnaires en français. Chaque participant a pris part à deux séances

7. L'adbésion à la culture des gangs de rue: son rôle dans l'identification des membres et son effet sur la délinquance (Fredette, 2014), Facteurs spécifiques et facteurs génériques: l'évaluation du risque et la prédiction de la récidive chez les délinquants associés aux gangs de rue (Guay et al., 2015) et Évaluer pour prévenir : les caractéristiques de la personnalité et les risques pris par les jeunes contrevenants associés aux gangs de rue (Laurier, Guay, Lafortune et Toupin, 2015). 
d'environ trois heures pour remplir les questionnaires et a reçu une compensation de trente dollars par rencontre.

\section{Variables et instruments}

Identité etbnique. Les participants ont rempli le Multigroup Ethnic Identity Measure - Revised (traduction libre québécoise) (MEIM-R: Phinney et Ong, 2007), constitué de 6 items sur une échelle de Likert en cinq points, allant de «Fortement en désaccord (1)» à «Fortement en accord (5)». Le MEIM-R est constitué de deux sous-dimensions étroitement liées au développement de l'identité ethnique: soit l'engagement (3 items) et l'exploration (3 items). Le score total obtenu varie de 6 à 30 pour le niveau d'identité ethnique et de 3 à 15 pour les souséchelles engagement et exploration. La sous-échelle engagement fait référence à l'intensité du sentiment d'appartenance envers un groupe ethnique et évalue le sentiment de fierté et les émotions positives par rapport à son ethnicité (p. ex. : «Je suis fortement attaché à mon groupe ethnique»). La sous-échelle exploration évalue le processus d'exploration lié aux activités concrètes effectuées pour mieux saisir et comprendre son ethnicité (p. ex.: «J'ai fréquemment fait des choses qui m’ont aidé à mieux comprendre mes origines ethniques»). Les scores totaux aux sous-échelles exploration et engagement ( $\alpha=0,81$ et 0,83 dans cet échantillon) peuvent être analysés séparément ou conjointement pour obtenir un score d'identité ethnique global ( $\alpha=0,81$ dans cet échantillon). Les alphas de Cronbach du MEIM-R dans cet échantillon révèlent une bonne cohérence interne des items liés au construit de l'identité et à ses sous-dimensions, tout comme lors de l'étude de validation du MEIM-R (Phinney et Ong, 2007).

Informations ethnoculturelles. Un questionnaire sociodémographique a permis d'obtenir les renseignements sur le pays de naissance du participant. La variable minorités racisées a été inspirée en partie des critères de la Loi sur l'équité en matière d'emploi (Gouvernement du Canada, 1995) pour définir les personnes issues d'un groupe de minorités visibles. Dans le cadre de cette étude, ont été considérées «les personnes qui ne sont pas de race blanche ou qui n'ont pas la peau blanche», soit les personnes qui font partie des groupes considérés comme minorités visibles comme les Asiatiques, les Noirs, les Arabes et les LatinoAméricains. Au Québec, une étude portant sur les trajectoires des adolescents contrevenants dans le système de justice pénale utilise 
également la catégorisation «Blanc» et «non-Blanc» afin d'établir des comparaisons selon les caractéristiques ethniques des participants (Lafortune et al., 2015).

Association autorévélée à un gang et adhésion à la culture de gang. Afin de déterminer si le participant s'identifie lui-même comme appartenant à un gang de rue ou non, une mesure autorévélée a été utilisée à partir d'une adaptation maison du questionnaire Gang Involvement Scale (GIS) de Spergel, Wa et Sosa (2005). Les questions suivantes: «Vous considérez-vous comme un membre d'un gang de rue?» et «Vous êtes-vous déjà considéré comme un membre de gang de rue?» ont permis de déterminer l'association autorévélée aux gangs de rue. Le participant a été identifié comme membre d'un gang à la suite d'une réponse positive à l'une des deux questions. L'autorévélation est une technique de recherche répandue, dont la fidélité et la validité sont adéquates, lorsqu'il est question de mesurer l'association à un gang de rue (Thornberry et al., 2003). En ce qui a trait à la mesure de l'adhésion du contrevenant à la culture de gang, les informations ont été recueillies à partir de la seconde échelle de la Mesure d'adhésion à la culture de gang $\left(\mathrm{MAC}^{\mathrm{g}}\right)$ (Fredette, 2014). Ce questionnaire a été construit, entres autres, à partir d'informations obtenues lors de groupes de discussion avec des jeunes contrevenants qui se disaient membres de gangs. Cette sous-échelle de 38 items est constituée de trois dimensions: l'adhésion du contrevenant aux symboles et aux signes de reconnaissance (10 items), aux règles et aux rituels (17 items) et aux normes et aux valeurs (11 items). Chaque item est coté sur une échelle de type Likert allant de «Pas du tout ou jamais (1)» à «Très, tout à fait ou tous les jours (5)». La sommation des items permet d'obtenir un score total qui varie de 38 à 190. La MACg a été conçue pour être utilisée auprès de jeunes contrevenants de 14 ans et plus, peu importe s'ils se considèrent comme membres d'un gang de rue ou non. Les alphas de Cronbach lors de l'étude préliminaire de la validation de l'instrument révèlent une bonne cohérence interne des items liés au construit de la culture de gang $(\alpha=0,95)$, ainsi qu'aux sous-échelles signes et symboles $(\alpha=0,88)$, règles et rituels $(\alpha=0,90)$ et normes et valeurs $(\alpha=0,80)$ (Fredette et Guay, 2014). Des alphas de Cronbach similaires ont été obtenus pour l'échantillon de la présente étude, soit 0,95 pour l'adhésion globale du contrevenant à la culture de gang et 0,88 pour la sous-échelle signes et symboles, 0,90 pour celle règles et rituels et 0,78 pour celle normes et valeurs. 
Les comportements délinquants autorévélés. Les participants ont rempli l'adaptation francophone du Self-Report of Offending - Revised (SRO-R; Huizinga, Esbenson et Weihar, 1991) afin de mesurer leurs comportements délinquants autorévélés lors des douze derniers mois avant leur prise en charge sous la LSJPA. Des études portant sur des clientèles délinquantes mineures ont permis de valider et de justifier l'utilisation de cet instrument auprès de cette population (Knight, Little, Losoya et Mulvey, 2004; Piquero, Macintosh et Hickman, 2002). Lorsqu'il est question de mesurer la délinquance, l'utilisation de questionnaires autorévélés a montré sa pertinence (Thornberry et Krohn, 2000).

\section{Résultats}

Portrait ethnoculturel des jeunes contrevenants de l'échantillon

Des analyses descriptives ont permis de dresser un portrait des caractéristiques ethnoculturelles des jeunes contrevenants de la présente étude (Tableau 1). L'échantillon est constitué principalement de jeunes contrevenants nés au Québec (72,5\%), suivi de ceux nés aux Antilles et sur le continent africain. En ce qui a trait à la génération d'immigration, $27,5 \%$ des jeunes ont immigré au Québec, $35 \%$ sont issus de la deuxième génération d'immigration (le participant né au Québec et au moins un parent né à l'étranger) et $37 \%$ sont issus de la troisième génération d'immigration ou plus (le participant et ses parents sont nés au Québec). Un peu plus de la moitié des jeunes contrevenants (53,6\%) font partie d'un groupe de minorités racisées.

\section{Identité ethnique et gang de rue}

Afin de déterminer s'il existe un lien entre le niveau d'identité ethnique et l'adhésion à la culture de gang, des analyses de corrélations simples ont été effectuées (Tableau 2). 
TA B LEA U 1

Répartition des jeunes contrevenants selon le pays de naissance, la génération d'immigration et le fait de faire partie d'un groupe de minorités racisées

\begin{tabular}{|c|c|c|}
\hline & \multicolumn{2}{|c|}{ Jeunes contrevenants } \\
\hline Pays de naissance & $n$ & $\%$ \\
\hline \multicolumn{3}{|l|}{ Amérique du Nord } \\
\hline Canada (Québec) & 50 & 72,5 \\
\hline États-Unis & 1 & 1,4 \\
\hline Mexique & 1 & 1,4 \\
\hline \multicolumn{3}{|l|}{ Antilles } \\
\hline Haïti & 4 & 5,8 \\
\hline République dominicaine & 1 & 1,4 \\
\hline \multicolumn{3}{|l|}{ Amérique du Sud } \\
\hline Colombie & 1 & 1,4 \\
\hline \multicolumn{3}{|l|}{ Europe } \\
\hline Biélorussie & 1 & 1,4 \\
\hline France & 2 & 2,9 \\
\hline \multicolumn{3}{|l|}{ Afrique } \\
\hline Algérie & 1 & 1,4 \\
\hline Angola & 1 & 1,4 \\
\hline Guinée & 1 & 1,4 \\
\hline Madagascar & 1 & 1,4 \\
\hline Sénégal & 1 & 1,4 \\
\hline \multicolumn{3}{|l|}{ Asie } \\
\hline Inde & 1 & 1,4 \\
\hline Irak & 1 & 1,4 \\
\hline Donnée non fournie & 1 & 1,4 \\
\hline \multicolumn{3}{|l|}{ Génération d'immigration } \\
\hline Première & 19 & 27,5 \\
\hline Deuxième & 24 & 34,8 \\
\hline Troisième et plus & 26 & 37,7 \\
\hline Minorités racisées & 37 & 53,6 \\
\hline
\end{tabular}


TABLEA U 2

Corrélations entre le niveau d'identité ethnique et le degré d'adhésion à une culture de gang par le jeune contrevenant $(n=69)$

\begin{tabular}{|l|c|c|c|c|c|c|}
\hline Variable & 2 & 3 & 4 & 5 & 6 & 7 \\
\hline 1. MEIM-R - Identité ethnique & $0,90^{* *}$ & $0,90^{* *}$ & $0,29^{*}$ & $0,26^{*}$ & $0,31^{*}$ & $0,25^{*}$ \\
\hline 2. MEIM-R - Exploration & & $0,62^{* *}$ & $0,33^{* *}$ & $0,32^{* *}$ & $0,34^{* *}$ & $0,27^{*}$ \\
\hline 3. MEIM-R - Engagement & & & 0,19 & 0,14 & 0,22 & 0,17 \\
\hline 4. MAC - Adhésion culture & & & & $0,88^{* *}$ & $0,97^{* *}$ & $0,88^{* *}$ \\
\hline 5. MACg- Signes et symboles & & & & & $0,80^{* *}$ & $0,64^{* *}$ \\
\hline 6. MACg - Règles et rituels & & & & & & $0,84^{* *}$ \\
\hline 7. MACg- Normes et valeurs & & & & & & \\
\hline
\end{tabular}

${ }^{*} p<0,05 .{ }^{* *} p<0,01$

Le niveau d'identité ethnique global est lié, et de manière positive, à l'adhésion à la culture de gang $(r=0,29, p=0,016)$. Ainsi, plus le niveau d'identité ethnique augmente, plus le niveau d'adhésion à la culture de gang augmente. Ces résultats pourraient s'expliquer, en partie du moins, par un lien plus étroit entre la dimension exploration de l'identité ethnique et l'adhésion globale à une culture de gang. En effet, seule la dimension exploration de l'identité ethnique est liée positivement au niveau d'adhésion à la culture de gang $(r=0,33, p=0,006)$, ainsi qu'aux trois sous-dimensions de l'adhésion à la culture de gang.

Des comparaisons de moyenne du niveau d'identité ethnique entre les jeunes contrevenants qui se considèrent comme membres d'un gang de rue et ceux qui ne se considèrent pas comme tels ont été réalisées. Les niveaux d'adhésion des jeunes contrevenants à la culture de gang ont également été comparés selon qu'ils ont révélé ou non être associés à un gang de rue. Les résultats sont présentés au Tableau 3.

En ce qui concerne le niveau d'identité ethnique global et les sousdimensions exploration et engagement, aucune différence significative n'est observée entre les jeunes contrevenants qui se révèlent être membres ou non de gang de rue. Le niveau d'adhésion global à une culture de gang, comme mesuré par la $\mathrm{MAC}^{\mathrm{g}}$, révèle que les jeunes contrevenants qui se considèrent comme membres de gang obtiennent des scores significativement plus élevés à la mesure d'adhésion globale à la culture de gang $(M=131,88)$, ainsi qu'à l'ensemble des sous-échelles de l'instrument, comparativement à ceux qui ne se considèrent pas comme membres de gang $(M=85,49)(t(66)=-9,71 ; p=0,000)$. Ainsi, se 
TA B LE A U 3

Comparaison du niveau d'identité ethnique et de l'adhésion à une culture de gang par les jeunes contrevenants membres et non membres d'un gang de rue

\begin{tabular}{|c|c|c|c|c|c|}
\hline \multirow[b]{2}{*}{ Score au MEIM-R } & \multicolumn{2}{|c|}{$\begin{array}{c}\text { Membre GDR } \\
(n=24)\end{array}$} & \multicolumn{2}{|c|}{$\begin{array}{c}\text { Non-membre GDR } \\
(n=45)\end{array}$} & \multirow[b]{2}{*}{$t$} \\
\hline & $M$ & $\dot{E} T$ & $M$ & 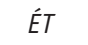 & \\
\hline Identité ethnique globale & 22,25 & 4,93 & 20,67 & 5,87 & $-1,13$ \\
\hline Exploration & 10,67 & 2,79 & 9,51 & 3,31 & $-1,46$ \\
\hline Engagement & 11,58 & 2,89 & 11,16 & 3,13 & $-0,55$ \\
\hline Score à la $M A^{g}$ & $M$ & ÉT & $M$ & $\dot{E} T$ & $t$ \\
\hline Adhésion globale & 131,88 & 14,85 & 85,49 & 24,75 & $-9,71^{* * *}$ \\
\hline Signes et symboles & 3,08 & 0,73 & 1,64 & 0,69 & $-8,12^{* * *}$ \\
\hline Règles et rituels & 3,70 & 0,43 & 2,38 & 0,77 & $-9,16^{* * *}$ \\
\hline Normes et valeurs & 3,49 & 0,53 & 2,64 & 0,63 & $-5,60 * * *$ \\
\hline
\end{tabular}

Note: $\mathrm{GDR}=\mathrm{Gang}$ de rue.

${ }^{* * *} p<0,001$.

considérer comme membre d'un gang de rue a un effet positif sur l'adhésion du contrevenant à la culture de gang.

Minorités racisées et gang de rue

Des analyses de comparaison de groupes ont été effectuées (tests de comparaison de moyennes et tableaux croisés) afin de s'assurer que les jeunes contrevenants qui font partie du groupe minorités racisées et ceux $\mathrm{du}$ groupe non-minorités racisées sont comparables sur la base de leurs caractéristiques de prise en charge et de leurs délits. Les résultats indiquent qu'ils sont comparables par rapport à leur âge $(t(67)=0,02$; $p=0,983)$, à la durée totale de leur sentence $(t(52)=0,76 ; p=0,449)$ et à la nature de leur prise en charge (garde ou communauté) $\left(\chi^{2}(1)=\right.$ $1,26 ; p=0,262)$. En ce qui a trait aux principaux paramètres de la délinquance, les deux groupes ne se différencient pas non plus. Ils sont comparables en ce qui a trait à l'âge au premier délit autorapporté $(t(67)$ $=-1,59 ; p=0,117)$, à la fréquence des crimes autorapportés $(Z=$ $-0,141, p=0,888)$, à la diversité $(t(67)=0,53 ; p=0,595)$ et à la gravité moyenne $(Z=-0,319, p=0,749)$ des délits autorapportés.

Afin de déterminer la présence d'un lien entre le fait de se considérer comme membre d'un gang et faire partie d'un groupe de minorités racisées, un test du $\chi^{2}$ d'indépendance a été réalisé. Les résultats n'in- 
diquent aucune différence significative dans l'association à un gang de rue selon le fait de faire partie d'un groupe de minorités racisées $\left(\chi^{2}(1\right.$, $N=69)=1,17, p=0,280)$. Dans cet échantillon, il n'est donc pas possible de conclure à un lien entre faire partie d'un groupe de minorités racisées et se reconnaître membre d'un gang de rue.

En ce qui concerne le niveau d'adhésion à la culture de gang $\left(\mathrm{MAC}^{\mathrm{g}}\right)$ (et à ses sous-dimensions signes et symboles, règles et rituels et normes et valeurs) par les jeunes contrevenants, aucune différence significative n'est observée entre les jeunes contrevenants du groupe minorités racisées et ceux du groupe non-minorités racisées comme l'indique le test de Student pour deux groupes indépendants $(t(67)=-1,34 ; p=0,184)$ (Tableau 4). Ainsi, il n'est pas possible de conclure a un effet entre l'adhésion du contrevenant à la culture de gang et faire partie d'un groupe de minorités racisées.

\section{TA B LEA U 4}

Comparaison du niveau d'identité ethnique et de l'adhésion à la culture de gang des jeunes contrevenants issus d'une minorité racisée et ceux qui n'en sont pas issus

\begin{tabular}{|c|c|c|c|c|c|}
\hline \multirow[b]{2}{*}{ Score au MEIM-R } & \multicolumn{2}{|c|}{$\begin{array}{l}\text { Minorités } \\
\text { racisées } \\
(n=37) \\
\end{array}$} & \multicolumn{2}{|c|}{$\begin{array}{c}\text { Non-minorités } \\
\text { racisées } \\
(n=32)\end{array}$} & \multirow[b]{2}{*}{$t$} \\
\hline & $M$ & $E ́ T$ & $M$ & 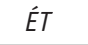 & \\
\hline Identité ethnique globale & 23,03 & 5,07 & 19,13 & 5,48 & $-3,07^{* *}$ \\
\hline Exploration & 10,95 & 2,81 & 8,72 & 3,17 & $-3,09$ ** \\
\hline Engagement & 12,08 & 2,66 & 10,40 & 3,23 & $-2,36^{*}$ \\
\hline Score à la $\mathrm{MAC}^{g}$ & $M$ & $E ́$ & $M$ & $E ́ T$ & $t$ \\
\hline Adhésion globale & 106,27 & 28,56 & 96,25 & 33,41 & $-1,34$ \\
\hline Signes et symboles & 2,30 & 0,89 & 1,96 & 1,06 & $-1,47$ \\
\hline Règles et rituels & 2,94 & 0,84 & 2,72 & 1,01 & $-0,98$ \\
\hline Normes et valeurs & 3,07 & 0,66 & 2,78 & 0,77 & $-1,67$ \\
\hline
\end{tabular}

Note: $\mathrm{GDR}=\mathrm{Gang}$ de rue.

${ }^{*} p<0,05$. ${ }^{* *} p<0,01$.

\section{Minorités racisées et identité ethnique}

Des comparaisons de moyennes du niveau d'identité ethnique (incluant les sous-dimensions engagement et exploration) entre les jeunes du groupe minorités racisées et ceux du groupe non-minorités racisées ont été effectuées 
TABLEA U 5

Régressions linéaires multiples et logistiques de l'association à un gang de rue et de l'adhésion à la culture de gang en fonction des caractéristiques personnelles (exploration, engagement, minorités racisées et âge)

\begin{tabular}{|l|c|c|c|c|c|c|}
\hline & \multicolumn{2}{|c|}{$\begin{array}{c}\text { Signes et } \\
\text { symboles }\end{array}$} & \multicolumn{2}{c|}{$\begin{array}{c}\text { Règles et } \\
\text { rituels }\end{array}$} & \multicolumn{2}{c|}{$\begin{array}{c}\text { Normes et } \\
\text { valeurs }\end{array}$} \\
\hline Variables & $B$ & Bêta & $B$ & Bêta & $B$ & Bêta \\
\hline Exploration & $0,12^{*}$ & 0,38 & $0,10^{*}$ & 0,35 & 0,06 & 0,25 \\
\hline Engagement & $-0,02$ & $-0,07$ & 0,02 & 0,05 & 0,01 & 0,02 \\
\hline Minorités racisées & 0,12 & 0,06 & $-0,04$ & $-0,02$ & 0,15 & 0,11 \\
\hline Âge & $-0,14$ & $-0,17$ & $-0,12$ & $-0,15$ & $-0,07$ & $-0,11$ \\
\hline \multirow{2}{*}{$R^{2}$} & \multicolumn{2}{|c|}{0,14} & \multicolumn{2}{c|}{0,14} & \multicolumn{2}{c|}{0,10} \\
\hline$F$ & \multicolumn{2}{|c|}{$2,59^{*}$} & \multicolumn{2}{c|}{$2,51^{*}$} & \multicolumn{2}{c|}{1,73} \\
\hline$n$ & \multicolumn{2}{|c|}{69} & \multicolumn{2}{c|}{69} & \multicolumn{2}{c|}{69} \\
\hline
\end{tabular}

\begin{tabular}{|c|c|c|c|c|c|}
\hline & \multicolumn{2}{|c|}{ Adhésion culture } & \multicolumn{3}{|c|}{ Membre GDR } \\
\hline Variables & $B$ & Bêta & $B$ & $\begin{array}{c}\text { Statistique } \\
\text { de Wald }\end{array}$ & $\begin{array}{l}\text { Rapport } \\
\text { de cote }\end{array}$ \\
\hline Exploration & $3,45^{*}$ & 0,35 & 0,14 & 1,49 & 1,15 \\
\hline Engagement & 0,05 & 0,01 & $-0,06$ & 0,23 & 0,95 \\
\hline Minorités racisées & 2,23 & 0,04 & 0,37 & 0,43 & 1,44 \\
\hline Âge & $-4,0$ & $-0,15$ & $-0,02$ & 0,00 & 0,99 \\
\hline$R^{2}$ & \multicolumn{2}{|c|}{0,13} & \multicolumn{3}{|c|}{0,06} \\
\hline$F / X^{2}$ & \multicolumn{2}{|c|}{$2,45^{\dagger}$} & \multicolumn{3}{|c|}{2,80} \\
\hline$\%$ de bonne classification & \multicolumn{2}{|c|}{-} & \multicolumn{3}{|c|}{65,2} \\
\hline$n$ & \multicolumn{2}{|c|}{69} & \multicolumn{3}{|c|}{69} \\
\hline
\end{tabular}

${ }^{\dagger} p<0,10 .{ }^{*} p<0,05$.

à l'aide de tests de Student pour deux groupes indépendants (Tableau 5). Les jeunes contrevenants du groupe minorités racisées ont rapporté des scores totaux significativement plus élevés au MEIM-R $(t(67)=-3,07$; $p=0,003)$ et aux sous-échelles exploration $(t(67)=-3,09 ; p=0,003)$ et engagement $(t(67)=-2,36 ; p=0,021)$, que ceux du groupe nonminorités racisées.

Identité ethnique, minorités racisées et gang de rue

La prochaine section examinera les facteurs associés à l'association autorévélée aux gangs et à l'adhésion à la culture de gangs. Les prédic- 
teurs sélectionnés pour les analyses de régression effectuées sont les scores obtenus aux sous-échelles exploration et engagement du MEIM-R, le fait de faire partie du groupe minorités racisées et l'âge des jeunes contrevenants (Tableau 5).

La régression logistique effectuée pour prédire l'association autorévélée à un gang de rue révèle que les scores obtenus au MEIM-R (sous-échelles engagement et exploration), le fait de faire partie du groupe minorités racisées et l'âge du jeune contrevenant ne sont pas statistiquement associés au fait de se reconnaître comme membre d'un gang $\left(\chi^{2}=\right.$ 2,79, $p=0,592$ ).

Les résultats des analyses de régressions linéaires multiples semblent indiquer que les scores obtenus à la sous-échelle exploration du MEIM-R sont associés aux scores obtenus aux sous-dimensions signes et symboles $(F(4,64)=2,59, p=0,045)$, et règles et rituels $(F(4,64)=2,52$, $p=0,050)$ de la $\mathrm{MAC}^{\mathrm{g}}$, et qu'ils permettent d'expliquer la variance dans les mêmes proportions $\left(R^{2}=0,14\right)$. Plus un jeune contrevenant présente des scores élevés à la sous-échelle exploration, plus ses scores sont aussi élevés aux sous-échelles signes et symboles $(B=0,12, p=0,015)$ et règles et rituels $(B=0,10, p=0,025)$ de la $\mathrm{MAC}^{\mathrm{g}}$.

Pour ce qui est de l'adhésion globale à une culture de gang par le jeune contrevenant, les résultats laissent supposer une tendance qui va dans le même sens que pour les sous-échelles signes et symboles et règles et rituels $(F(4,64)=2,45, p=0,055)$. En effet, plus un jeune contrevenant rapporte des scores élevés à la sous-échelle exploration du MEIM-R, plus son score total à la $\mathrm{MAC}^{\mathrm{g}}$ a tendance à être élevé $(B=3,45$, $p=0,026)$. Ce modèle expliquerait $13 \%$ de la variance du niveau d'adhésion à la culture de gang.

\section{Discussion}

Minorités racisées, identité ethnique et gang de rue: qu'en est-il pour les jeunes contrevenants de la région montréalaise et ses environs?

Les résultats aux premières analyses ont révélé que les jeunes contrevenants qui se reconnaissent en tant que membres d'un gang de rue adhèrent davantage à la culture de gang, telle que l'a définie Fredette (2014). Ces résultats concordent avec ceux obtenus dans ses travaux de recherche qui incluaient également des contrevenants adultes. Au sein 
du présent échantillon, il y a donc un lien entre se reconnaître dans un gang de rue et adhérer à la culture de gang.

Les jeunes de l'échantillon faisant partie d'un groupe de minorités racisées présentaient une identité ethnique globale plus élevée que ceux issus de la majorité raciale blanche, tout comme dans l'étude de Guitart (2011). Le sentiment d'être différents des jeunes de la majorité raciale amène les jeunes contrevenants n'en faisant pas partie à entreprendre davantage de démarches et à avoir un sentiment d'appartenance envers leurs origines ethniques plus élevé que les jeunes contrevenants issus de la majorité raciale. Des analyses plus approfondies ont révélé que se considérer comme membre d'un gang de rue et adhérer à la culture de gang ne sont pas le propre des personnes issues d'un groupe de minorités racisées. Dans cet échantillon, autant de jeunes contrevenants faisant partie de la majorité raciale se reconnaissent en tant que membres de gang et adhèrent au même degré à la culture de gang de rue que ceux qui font partie des minorités racisées. Ces résultats vont à l'encontre des aprioris et également de ce qui est véhiculé dans les écrits scientifiques. Pris en considération conjointement, ni l'âge du jeune contrevenant, ni le fait qu'il fait partie d'un groupe de minorités racisées, ni son niveau d'identité ethnique n'ont permis de prédire s'il se considérait comme membre d'un gang de rue. Des analyses plus approfondies permettent d'avancer que ce sont les démarches ou les activités pour mieux comprendre et connaître d'où ils viennent (la dimension exploration de l'identité ethnique) qui permettent le mieux de prédire la tendance à adhérer à la culture de gang, et ce, peu importe l'âge ou le fait d'appartenir à un groupe de minorités racisées. Les effets étaient plus prononcés lorsqu'il était question d'adhérer aux signes et aux symboles, ainsi qu'aux règles et rituels de la culture de gang. Tel qu'abordé par Umana-Taylor et ses collaborateurs (2014), il est possible de croire que s'associer à un gang de rue pourrait servir de vecteur à explorer son identité ethnique en côtoyant d'autres jeunes semblables sur le plan culturel ou ethnique. Ces résultats appuient le constat de Fredette et Guay (2014), comme quoi l'association à un gang de rue semble plus liée à une question d'intensité, plutôt que de nature, soit être ou ne pas être dans un gang de rue.

En résumé, ce qui semble importer lorsqu'il est question d'adhérer à la culture de gang n'est pas qu'un jeune contrevenant se considère comme membre d'un gang de rue ou non, ou qu'il fasse partie d'un groupe de minorités racisées ou non, mais plutôt où il se situe sur le 
plan de l'exploration de ses origines ethniques. Plus il explore ses origines ethniques, plus il a tendance à adhérer à la culture de gang.

Une des principales forces de cette étude est de ne pas se limiter à une mesure dichotomique de l'association à un gang de rue et de l'identité ethnique, et d'avoir inclus des mesures permettant plus de nuances pour évaluer l'identité ethnique et l'adhésion à la culture de gang. À notre connaissance, elle représente également la première étude quantitative qui explore les liens entre l'identité ethnique et la culture de gang auprès de jeunes contrevenants. Cette démarche exploratoire a permis d'avoir un portrait, quoique parcellaire, des liens entre l'identité ethnique et la culture de gang afin de cibler dans des recherches futures des pistes pour mieux cerner la complexité du développement identitaire chez les jeunes contrevenants.

Parmi les limites, il est à noter que compte tenu du manque d'informations sur la composition ethnique des gangs auxquels les jeunes ont rapporté s'associer, on ne peut pas affirmer que s'identifier à un gang de rue pourrait servir de vecteur à explorer une identité ethnique spécifique en côtoyant d'autres jeunes de la même origine ethnique, mais simplement de souligner un processus identitaire commun par le regroupement avec des pairs vivant des expériences semblables.

Dans des études ultérieures, il serait intéressant et pertinent d'approfondir la question de l'exploration de l'identité ethnique par l'entremise d'entrevues qualitatives en vue de préciser quelles étaient les activités, les démarches que les jeunes ont entreprises ou les personnes avec lesquelles ils sont entrés en contact afin de mieux connaître et explorer leurs origines. Cela permettrait également de départager les activités culturelles ou ethniques entreprises par l'entremise des activités du gang de celles au sein de la communauté ou de l'entourage des jeunes. Le réseau social des délinquants est souvent restreint à d'autres pairs délinquants, particulièrement à l'adolescence où les jeunes rejettent les activités de la sphère familiale (Fredette, 2014).

De plus, le développement de l'identité ethnique étant un processus dynamique, l'utilisation d'un seul temps de mesure a pu influencer les résultats. Il serait pertinent dans les recherches futures de mettre en place des devis de recherche longitudinaux pour mieux appréhender le processus identitaire.

Par ailleurs, le fait que la majorité des jeunes de l'échantillon étaient placés en centre de réadaptation au moment de participer à la recherche a pu influencer leurs réponses aux différents questionnaires traitant de 
la question identitaire ou culturelle. Les centres de réadaptation représentent une «culture» en soi, qui s'appuie fondamentalement sur le respect des règles et de l'autorité. Ces lieux s'accompagnent d'une proscription générale interdisant tout autre comportement que celui qui est prescrit dans le cadre (Goffman, 1968). Dans ce contexte, il peut être difficile pour un jeune de se positionner quant à ses «identités» ou «cultures», souvent multiples, ayant une seule culture de référence dans l'environnement immédiat, celle du centre de réadaptation, qui laisse peu de place à l'individualité et à la différenciation.

Il importe enfin de mentionner que ces résultats ne peuvent pas être généralisés aux jeunes non délinquants ou à l'ensemble des jeunes contrevenants. En effet, cet échantillon n'est pas représentatif de la majorité des jeunes contrevenants, compte tenu du niveau élevé de leur délinquance (nature, fréquence et gravité des délits commis). Toutefois, à la lumière de ces résultats, il est possible de s'interroger sur la manifestation des démarches d'exploration de l'identité ethnique chez les adolescents non délinquants. Ces deniers ont-ils tendance à adhérer à la culture d'un autre groupe de jeunes semblables, mais non criminalisés, comme une équipe de sport ou un club de musique ou de danse?

En conclusion, le fait que les jeunes en période d'exploration de leurs origines ethniques ont tendance à adhérer davantage à la culture de gang met en avant-plan la complexité des liens que peuvent avoir les jeunes envers leur ethnicité. Ce constat fait ressortir l'importance de s'intéresser à ces questions identitaires lors d'interventions auprès de jeunes contrevenants, et ce, peu importe leurs origines.

\section{Références}

Agnew, R. (1992). Foundation for a general strain theory of crime and delinquency. Criminology, 30(1), 47-88.

Bolaffi, G., Bracalenti, R., Braham, P. H. et Gindro, S. (2003). Dictionary of Race, Ethnicity and Culture. Londres, Royaume-Uni: Sage Publications.

Chatterjee, J. (2006). Un rapport de recherche sur les gangs de jeunes: problèmes, perspectives et priorités. Ottawa, Ontario: Sous-direction de la recherche et de l'évaluation, Direction des services de police communautaires, contractuels et autochtones, Gendarmerie royale du Canada.

Chettleburgh, M. C. (2003). Results of the 2002 Canadian policy survey on youth gangs. Ottawa, Ontario: Sécurité publique du Canada.

Cohen, A. K. (1955). Delinquent boys: The culture of the gang. Glencoe, IL: Free Press.

De laco, G. A. (2006). Juvenile street gang members and ethnic identity in Montreal (Thèse de doctorat). Université McGill. 
Egley, A. Jr., Howell, J. C. et Major, A. K. (2006). National Youth Gang Survey: 1999-2001. Washington, DC: U.S. Department of Justice, Office of Justice Programs, Office of Juvenile Justice and Delinquency Prevention.

Erikson, E. H. (1972). Adolescence et crise: La quête de l'identité (J. Nass et C. Louis-Combet, trad.). Paris, France: Flammarion.

Esbensen, F.-A. et Carson, D. C. (2012). Who are the gangsters? An examination of the age, race/ethnicity, sex, and immigration status of self-reported gang members in a seven-city study of American youth. Journal of Contemporary Criminal Justice, 28(4), 465-481.

Esbensen, F. A. et Winfree, L. T. (1998). Race and gender differences between gang and nongang youths: Results form a multisite survey. Justice Quarterly, 15(3), 505-526.

Esbensen, F. A., Peterson, D., Taylor, T. J. et Freng, A. (2010). Youth violence: Sex and race differences in offending, victimization, and gang membership. Philadelphia, PA: Temple University Press.

Franzese, R. J., Covey, H. C. et Ménard, S. (2006). Youth gangs (3 éd.). Springfield, IL: Charles C. Thomas Publisher, Ltd.

Fredette, C. (2014). Mesurer l'apport unique de l'adbésion à la culture des gangs de rue à l'explication des conduites délinquantes chez les adolescents et les bommes contrevenants (Thèse de doctorat inédite). Université de Montréal.

Fredette, C. et Guay, J.-P. (2014). La culture de gang, l'identité de membre et la délinquance. Dans J.-P. Guay et C. Fredette (dir.), Le phénomène des gangs de rue: Théories, évaluations, interventions (p. 151-166). Montréal, Québec: Presses de l'Université de Montréal.

French, S. E., Kim, T. E. et Pillado, O. (2006). Ethnic identity, social group membership, and youth violence. Dans N. G. Guerra et E. P. Smith (dir.), Preventing youth violence in a multicultural society (p. 47-73). Washington, DC: American Psychological Association.

Freng, A. et Esbensen, F.-A. (2007). Race and gang affiliation: An examination of multiple marginality. Justice Quarterly, 24(4), 600-628.

Goffman, E. (1968). Asiles. Études sur la condition sociale des malades mentaux et autres reclus. Paris, France: Les éditions de Minuit.

Guay, J.-P., Laurier, C., Morselli, C., Renaud, P., Lafortune, D. et Joyal, C. (2015). Facteurs spécifiques et facteurs génériques: l'évaluation du risque et la prédiction de la récidive chez les délinquants associés aux gangs de rue (Rapport n 2011 - GR-139284). Québec: Fonds de recherche du Québec - Société et culture (FRQSC).

Guerra, N. G. et Smith, E. P. (2006). Preventing youth violence in a multicultural society. Washington, DC: American Psychological Association.

Guitart, M. E. (2011). Ethnic identity in an intercultural geography: An empirical study. Canadian Social Science, 7(2), 64-71.

Gouvernement du Canada (1995). Loi sur l'équité en matière d'emploi. Repéré à http://laws.justice.gc.ca/fra/lois/e-5.401/page-1.html\#h-3

Hagedorn, J. M. (2008). A World of Gangs: Armed Young Men and Gangsta Culture. Minneapolis, $\mathrm{MN}$ : University of Minnesota Press. 
Hamel, S., Alain, M., Messier-Newman, K., Domond, P. et Pagé, J.-P. (2013). Le phénomène des gangs de rue, ici et ailleurs: mise à jour des connaissances. TroisRivières, Québec: Université du Québec à Trois-Rivières.

Hill, K. G., Howell, J. C., Hawkins, J. D. et Battin-Pearson, S. R. (1999). Childhood risk factors for adolescent gang membership: Results from the Seattle Social Development Project. Journal of Research in Crime and Delinquency, 36(3), 300-322.

Huizinga, D., Esbenson, F. et Weihar, A. (1991). Are there multiple paths to delinquency?. Journal of Criminal Law and Criminology, 82(1), 83-118.

Klein, M. W. (1995). The American Street Gang: Its nature, prevalence, and control. Oxford, NY: Oxford University Press.

Klein, M. W. et Maxson, C. L. (2006). Street Gangs: Patterns and Policies. New York, NY: Oxford University Press.

Knight, G. P., Little, M., Losoya, S. H. et Mulvey, E. P. (2004). The self-report of offending among serious juvenile offenders: Cross-gender, cross-ethnic/ race measurement equivalence. Youth Violence and Juvenile Justice, 2, 273-295.

Knight, G. P., Losoya, S. H., Cho, Y. I., Chassin, L., Williams, J. L. et CotaRobles, S. (2012). Ethnic identity and offending trajectories among Mexican American juvenile offenders: Gang membership and psychosocial maturity. Journal of Research on Adolescence, 22(4), 782-796.

Lafortune, D., Royer, M. N., Rossi, C., Turcotte, M. E., Boivin, R., Cousineau, M.-M., ... Trépanier, J. (2015). La loi sur le système de justice pénale pour les adolescents sept ans plus tard: portrait des jeunes, des trajectoires et des pratiques (Rapport n² 2011 - TA-144097). Québec: Fonds de recherche du Québec - Société et culture (FRQSC).

Laurier, C., Guay, J.-P., Lafortune, D. et Toupin, J. (2015). Évaluer pour prévenir: les caractéristiques de la personnalité et les risques pris par les jeunes contrevenants associés aux gangs de rue (Rapport no 2011 - GR-138835). Québec: Fonds de recherche du Québec - Société et culture (FRQSC).

Gouvernement du Canada (2002). Loi sur le système de justice pénale pour les adolescents. Repéré à http://laws-lois.justice.gc.ca/fra/lois/y-1.5/

Mc Andrew, M., Ledent, J. et Bakhshaei, M. (2013). Des mots pour le dire: La persistance des identités, des appartenances et des processus d'exclusion. Montréal, Québec: Centre d'études ethniques des universités montréalaises.

Mock, D. (2005). Glossary of Terms. Toronto, Ontario: Fondation canadienne des relations raciales.

Perreault, M. et Bibeau, G. (2003). La Gang: une chimère à apprivoiser. Montréal, Québec: Les Éditions du Boréal.

Phinney, J. S. et Ong, A. D. (2007). Conceptualization and measurement of ethnic identity: Current status and future directions. Journal of Counselling Psychology, 54(3), 271-281.

Phinney, J. S. (1990). Ethnic identity in adolescents and adults: Review of research. Psychological Bulletin, 108(3), 499-514.

Phinney, J. S. (2008). La formation de l'identité culturelle dans les contextes multiculturels. Communication présentée à la conférence interdisciplinaire 
Generations in Flux: Ethnicity, Integration and Family Ties (octobre). Helsinki, Finlande: ETMU. Repéré à https://www.coe.int/t/dg4/youth/Source/ Resources/Forum21/II_Issue_No4/II_No4_Cultural_identity_fr.pdf.

Piquero, A. R., Macintosh, R. et Hickman, M. (2002). The validity of a selfreported delinquency scale: Comparisons across gender, age, race, and place of residence. Sociological Methods and Research, 30(4), 492-529.

Rivas-Drake, D., Seaton, E. K., Markstrom, C., Quintana, S., Syed, M., Lee, R. M. ... Yip, T. (2014). Ethnic and racial identity in Adolescence: Implications for psychosocial, academic, and health outcomes. Child development, 85(1), 40-57.

Sanchez-Jankowski, M. (1991). Islands in the Street: Gangs and American Urban Society. Berkeley, CA: University of California Press.

Sanchez-Jankowski, M. (2003). Gangs and social change. Theoretical Crimino$\log y, 7(2), 191-216$.

Savelli, L. (2004). Gangs across America and Their Symbols. New York, NY: Looseleaf Law Publications Inc.

Spergel, I. A., Wa, K. M., et Sosa, R. V. (2005). Evaluation of the BloomingtonNormal Comprehensive Gang Program. Chicago, IL: School of Social Service Administration.

Spergel, I. A. (2009). Gang databases: To be or not to be. Criminology and Public Policy, 8(4), 667-674.

Thornberry, T. P. et Krohn, M. D. (2000). The self-report method for measuring delinquency and crime. Dans D. Duffe, R. D. Crutchfield, S. Mastrofski, L. Mazerolle et D. McDowall (dir.), Criminal justice: Measurement and analysis of crime and justice (p. 33-83). Washington DC: National Institute of Justice.

Thornberry, T. P., Krohn, M. D., Lizotte, A. J., Smith, C. A. et Tobin, K. (2003). Gangs and Delinquency in Developmental Perspective. New York, NY: Cambridge University Press.

Umaña-Taylor, A. J., Quintana, S. M., Lee, R. M., Cross, W. E., Rivas-Drake, D., Schwartz, S. J. ... Seaton, E. (2014). Ethnic and racial identity during adolescence and into young adulthood: An integrated conceptualization. Child Development, 85(1), 21-39.

van Gemert, F., Peterson, D. et Lien, I.-L. (2008). Street gangs, migration and ethnicity. Portland, OR: Willan Publishing.

Vigil, J. D. (1988). Group processes and street identity: Adolescent Chicano gang members. Ethos, 16(4), 421-445.

Wortley, S. et Tanner, J. (2006). Immigration, social disadvantage and urban youth gangs: Results of a Toronto-Area survey. Canadian Journal of Urban Research, 15(2), 18-37.

Wortley, S. et Tanner, J. (2008). Respect, friendship, and racial injustice: Justifying membership in a Canadian City. Dans F. van Gemert, D. Peterson et I.-L. Lien (dir.), Street gangs, migration and ethnicity (p. 192-208). Portland, OR: Willan Publishing. 


\title{
Allegiance to gang culture: The role of ethnic identity among young offenders in the Montreal area
}

\begin{abstract}
The increased presence of ethnic minorities in youth centers in Quebec raises complex and difficult questions. This study explores the effect of ethnic identity and being part of a racialized minority group on offenders' self-reported gang association and allegiance to gang culture. Participants $(\mathrm{n}=69$; age 14-20 years) were recruited from youth rehabilitation centers in the Montreal area. The results suggest that there was no difference between young offenders from racialized minorities and those who were not in terms of association with gangs. Regression analysis indicates that the more a young offender reports an interest in exploring his own ethnic identity, the more commitment he shows to the signs and symbols as well as the rules and rituals aspects of gang culture, regardless of age or racial status. This study highlights the importance of focusing on the question of identity during interventions with young offenders, regardless of their origins.
\end{abstract}

KEYWORDS - Ethnic identity, minorities, ethnicity, juvenile delinquency, street gangs.

\section{La adhesión a la cultura de pandilla. El rol de la identidad étnica en los jóvenes delincuentes de Montreal y alrededores}

RESUMEN - La creciente presencia de jóvenes miembros de grupos etnoculturales minoritarios en las instituciones para jóvenes delincuentes en Quebec es una problemática compleja y preocupante. El presente estudio explora el rol de la identidad étnica $y$ del hecho de formar parte de un grupo minoritario racial en la asociación autorevelada a una pandilla y en la adhesión a la cultura pandillera. Los participantes ( $\mathrm{n}=69$; edad 14-20 años) han sido reclutados en los centros de readaptación de la región de Montreal. De los resultados se desprende que el hecho de reconocerse como miembro de una pandilla no difiere entre los jóvenes provenientes de las minorías raciales con respecto a aquéllos que no lo son. Los análisis de regresiones múltiples completados revelan que cuanto más elevado es el nivel de exploración de la identidad étnica del joven delincuente, mayor es la adhesion de este a las dimensiones signos y símbolos y reglas y rituales de la adhesión de la cultura pandillera, y esto último, a pesar de su edad, o de que forme parte de un grupo de minorías raciales. Este estudio resalta la importancia de interesarse a las cuestiones de identidad, cuando se interviene con jóvenes delincuentes, independientemente de sus orígenes.

PALABRAS CLAVE - Identidad étnica, minorías, etnicidad, delincuencia juvenil, pandillas. 